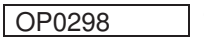

THE COMPARATIVE EFFECTIVENESS OF CYCLING TUMOURNECROSIS FACTOR INHIBITOR (TNFI) VERSUS SWAPPING TO A NONTNFI ON PATIENT-REPORTED FUNCTIONAL ABILITY OF PATIENTS WITH RHEUMATOID ARTHRITIS

M.A. Lopez-Olivo ${ }^{1}$, A. Matusevich ${ }^{1}$, S.B. Cantor ${ }^{2}$, G. Pratt ${ }^{3}$, M.E. Suarez-Almazor ${ }^{1}$. ${ }^{1}$ General Internal Medicine; ${ }^{2}$ Health Services Research; ${ }^{3}$ Research Medical Library, The University of Texas, MD Anderson Cancer Center, Houston, USA

Background: Data on patient-reported functional ability to evaluate the optimal strategy for patients who have failed to first TNFi is scarce. Patient-reported outcomes are a critical component of assessing whether clinicians are improving the wellbeing of patients.

Objectives: We conducted a systematic review and meta-analysis to evaluate the comparative effectiveness of two strategies, cycling versus swapping, on patient-reported functional ability and other patient-reported outcomes.

Methods: Four electronic databases were searched (MEDLINE, EMBASE, Cochrane Library, and Web of Sciences). Sources of grey literature (unpublished records) were searched through clinicaltrials.gov and other websites. The selection process, risk of bias assessment, and data extraction were performed by two independent reviewers. We included controlled trials evaluating patient-reported outcomes in patients either cycling to a second TNFi or swapping to a targeted drug with an alternative mechanism of action. Other outcomes reported included pain, patient global assessment, fatigue, and quality of life.

Results: We included 13 studies reporting data on 4394 patients. The reported cycling strategies were adalimumab, certolizumab, etanercept, golimumab, or infliximab; swapping strategies were abatacept, rituximab, tocilizumab, or tofacitinib. For the individual comparisons, TNFi versus disease modifying antirheumatic drug (DMARD), there was a statistically significant increase in functional ability from baseline to 14 weeks, favouring those patients receiving the cycling strategy (Mean Difference (MD) $-0.20,95 \% \mathrm{Cl}:-0.34$ to -0.06 ; scores ranging from 0 to 3). Differences favouring cycling when compared to a DMARD were also observed for pain, fatigue, and patient global assessment. Similarly, when comparing nonTNFi versus DMARD, there was a statistically significant increase in functional ability from baseline to 24 weeks, favouring those patients receiving the swapping strategy (MD $-0.31,95 \% \mathrm{Cl}:-0.35$ to -0.27 ; scores ranging from 0 to 3). Differences favouring cycling when compared to a DMARD were also observed for pain, sleep, fatigue, patient global, and quality of life (SF-36 physical and mental components). Three RCTs directly compared the two strategies. There was no statistically significant differences in the functional disability reported between those patients assigned to the cycling strategy compared with those assigned to the swapping strategy at $12,24,36$ or 52 weeks (MD at 52 weeks $-0.05,95 \% \mathrm{Cl}:-0.18$ to 0.09 ; score ranging from $0-3$ ).

Conclusions: Although evidence from previous reports suggest that swapping may be more effective than cycling when evaluating some clinical outcomes our results suggest that with the current evidence both strategies are equally effective in improving functional disability and other patient-reported outcomes.

Acknowledgements: Funding for this project was provided by the Rheumatology Research Foundation Investigator Award.

Disclosure of Interest: M. Lopez-Olivo Grant/research support from: Rheumatology Research Foundation, A. Matusevich Grant/research support from: Rheumatology Research Foundation, S. Cantor: None declared, G. Pratt: None declared, M. Suarez-Almazor: None declared

DOI: 10.1136/annrheumdis-2018-eular.6989

\section{OP0299 COST-EFFECTIVENESS OF TAPERING TNF BLOCKERS VERSUS CONVENTIONAL SYNTHETIC DMARDS IN RHEUMATOID ARTHRITIS: FIRST YEAR RESULTS OF THE RANDOMISED CONTROLLED TARA-STUDY}

E. van Mulligen ${ }^{1}$, A. E. A. M.Weel ${ }^{1,2}$, T.M. Kuijper ${ }^{2}$, J.J. Luime ${ }^{1}$, J.M.W. Hazes ${ }^{1}$, P. H.P. de Jong ${ }^{1,2},{ }^{1}$ Rheumatology, Erasmus MC; ${ }^{2}$ Rheumatology, Maasstad hospital, Rotterdam, Netherlands

Background: Current guidelines recommend to consider tapering treatment in rheumatoid arthritis (RA) patients who are in sustained remission, but the optimal approach to de-escalate conventional synthetic and biological DMARDs (respectively csDMARDs and bDMARDs) remains unknown. The benefits of tapering are a decreased risk of long-term adverse events and a reduction of health care costs, especially when bDMARDs are tapered. However, tapering treatment may lead to more transient or persistent disease flares, which have a direct impact on patients' lives and societal costs.

Objectives: The aim of this study is to evaluate the difference in cost-effectiveness of tapering csDMARD or anti-TNF therapy during one year of follow-up.

Methods: The TARA trial is a multicenter single-blinded randomised controlled trial. Included were RA patients that used a combination of csDMARDs and antiTNF and who were at least for 3 months in sustained remission, defined as a DAS $\leq 2.4$ and a swollen joint count $(\mathrm{SJC}) \leq 1$. Patients were randomised into gradual tapering csDMARDs followed by the TNF blocker or vice versa. Medication was tapered in three steps over the course of 6 months. Gradual tapering was done by cutting the dosage into half, a quarter and thereafter it was stopped. Data on QALYs (measured with the Dutch EuroQol [EQ5D]), direct, and indirect costs were used to calculate the Incremental Cost Effectiveness Ratio (ICER). Direct costs comprises costs for treatment and medical consumption, while indirect costs comprises costs due to loss of productivity (i.e. sick leave and unemployment). Results: A total of 187 patients were randomly assigned to tapering csDMARDs $(n=93)$ or tapering anti-TNF $(n=94)$. Patients had an average symptom duration of 6.7 years and were predominantly female $(66 \%)$ with an average age of 56.4 years (figure $1 \mathrm{~A}$ ). Average QALYs (SD), over 1 year, for tapering csDMARDs or anti-TNF were, respectively, $0.82(0.1)$ and $0.83(0.1)$ (figure 1B). One year after inclusion a none significant difference in cumulative flare ratio of $9 \%$ was observed (overall flare ratio $36 \%$ ). Patients in the anti-TNF tapering group had lower costs per QALY (SD) (€11390 (6809)) compared to patients in the csDMARD tapering group (€21 804 (8329)). The difference in costs per QALY were mainly determined by the medication costs (figure 1B). The Incremental Cost Effectiveness Ratio (ICER, 95\% Cl) between tapering csDMARDs and antiTNF was $€ 31922$ ( $€ 920572, € 984416)$ (figure 1C). Tapering anti-TNF was $>95 \%$ cost-effective across all willingness-to-pay thresholds compared to tapering csDMARDs (figure 1D).
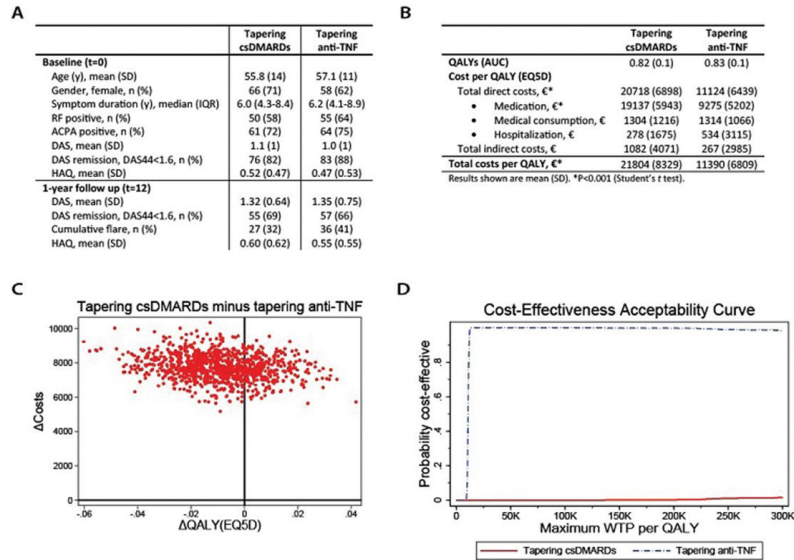

Abstract OP0299 - Figure 1 Summary of economic evaluation. (A) baseline characteristics and results after 12 months of follow-up of both tapering groups. (B) total costs per QALY after 1year of follow-up. (C) cost effectiveness and plane of costs of taoering csDMARDS minus costs for tapering anti-TNF. (D) acceptability curves for tapering csDMARDs and tapering anti-TNF. Abbreviations: AUC; area under the curve, csDMARDs; conventional synthetic DMARDs, EQ-5D; Dutch EuroQol, QALY; quality adjested life year, WTP willingness to pay.

Conclusions: Tapering anti-TNF is more cost-effective compared to tapering csDMARDs. Therefore, in RA patients who are in sustained remission we advise to taper anti-TNF first, but before tapering therapy rheumatologist should take the risk of a disease flare and patient's wishes into account.

Disclosure of Interest: None declared

DOI: 10.1136/annrheumdis-2018-eular.2051

\section{OP0300 REDUCING AVOIDABLE BIOLOGIC DRUG WASTAGE THROUGH COLLABORATION BETWEEN PATIENTS AND CARE PROVIDERS: THE LEEDS SPONDYLOARTHRITIS SERVICE EXPERIENCE}

A. Barr, D. Pickles, N. Fadl, J. Dou, C. Vandevelde, S. Dubash, J.E. Freeston, H. Marzo-Ortega. NIHR Leeds Biomedical Research Centre, University of Leeds. UK., Leeds Teaching Hospitals Trust and Leeds Institute of Rheumatic and Musculoskeletal Medicine, Leeds, UK

Background: The Leeds rheumatology department manages a cohort of approximately 4,000 patients with inflammatory arthritis receiving biologic therapies with an estimated annual cost of $£ 15,000,000$. Of these, approximately 1,000 have axial Spondyloarthritis or Psoriatic arthritis $(\mathrm{SpA})$. Biologic drug wastage with selfinjectable drugs can occur when patients have no further use for their existing stockpile of drug (typically occurring when receiving further stock around the time of stopping, pausing or switching drugs). Wastage is recorded by home-delivery companies on receiving returned 'unusable' stock. With intravenous drugs, was tage occurs when patients don't attend infusions. Reducing risk of self-injectable wastage has been achieved (1) but reducing self-injectable and intravenous biologic wastage has not. 